\title{
Efectividad de la vacuna anti-Influenza en embarazadas y lactantes
}

\author{
Effectiveness of Influenza vaccine in mothers and breastfeeding infants
}

Zaman K y col. N Engl J Med 2008; 359:1555-64

\section{Objetivo}

Determinar la seguridad y la inmunogenicidad de la vacuna anti-Neumococo y la efectividad clínica de la vacuna antiInfluenza aplicada en casos de influenza en embarazadas y lactantes.

\section{Diseño}

Ensayo clínico controlado aleatorizado y doble ciego, llevado a cabo en Bangladesh.

\section{Pacientes}

Fueron aleatorizadas 340 embarazadas durante el tercer trimestre y 331 neonatos. El seguimiento fue de 1651 personas/meses para los niños y de 2165 personas/meses para las madres.

Tabla 1: efectividad clínica de la vacuna anti-Influenza.

\section{Intervención}

Las embarazadas fueron aleatorizadas a cuatro grupos: las de los grupos 1 y 2 recibieron vacuna anti-Neumococo, y las de los grupos 3 y 4 , anti-Influenza. Los niños fueron aleatorizados a recibir vacuna anti-Neumococo o anti-Haemophilus, esta estrategia como parte del estudio primario.

\section{Medición de los resultados primarios}

Primer episodio de influenza en lactantes confirmado por laboratorio antes de las 24 semanas de vida. El análisis se realizo por intención de tratar.

\section{Resultados}

El dolor local luego de la vacunación fue significativamente más frecuente en las mujeres que recibieron vacuna anti-Neumococo $(11,4 \%$ vs $4,1 \% ; p<0,01)$. Los resultados de efectividad de la vacuna anti-Influenza se describen en la tabla 1.

\begin{tabular}{|c|c|c|c|c|c|}
\hline & & \multicolumn{2}{|c|}{ Episodios por grupo } & \multirow[b]{2}{*}{ Efectividad clínica (IC95\%) } & \multirow[b]{2}{*}{ Diferencia de riesgo (IC95\%) } \\
\hline & & Gontrol & Vacuna & & \\
\hline \multicolumn{2}{|c|}{ Lactantes por grupo (en personas meses) } & 870 & 881 & & \\
\hline \multirow{2}{*}{$\begin{array}{l}\text { Enfermedad } \\
\text { respiratoria febril }\end{array}$} & Cualquier temperatura & 153 & 110 & $28,9(6,9$ a 45,7$)$ & $-28,1(-48,2$ a $-8,0)$ \\
\hline & Más de $38^{\circ} \mathrm{C}$ & 77 & 56 & $28,1(-4,6$ a 50,6$)$ & $-13,7(-28,8$ a 0,5$)$ \\
\hline \multicolumn{2}{|c|}{ Visitas a la clínica } & 92 & 54 & $42.0(18.2$ a 58.8$)$ & $-24,5(-39,5$ a $-9,5)$ \\
\hline \multirow{2}{*}{$\begin{array}{l}\text { Prueba de } \\
\text { Influenza }\end{array}$} & Cualquier temperatura & 79 & 41 & $48,7(25,4$ a 64,7$)$ & $-24,4(-38,0$ a $-10,8)$ \\
\hline & Más de $38^{\circ} \mathrm{C}$ & 16 & 6 & $62,8(5,0$ a 85,4$)$ & $-6,4(-12,2$ a $-0,5)$ \\
\hline \multicolumn{2}{|c|}{ Madres por grupo (en personas/meses) } & 1076 & 1089 & & \\
\hline \multirow{2}{*}{$\begin{array}{c}\text { Enfermedad } \\
\text { respiratoria febril }\end{array}$} & Cualquier temperatura & 77 & 50 & $35,8(3,7$ a 57,2$)$ & $-14,2(-25,5$ a $-2,9)$ \\
\hline & Más de $38^{\circ} \mathrm{C}$ & 33 & 19 & $43,1(-9,0$ a 70,3$)$ & $-7,3(-14,5$ a $-0,1)$ \\
\hline
\end{tabular}

RRR: (reducción de riesgo relativo)

Por cada 100 embarazadas vacunadas se previnieron 14 episodios de enfermedad respiratoria febril en lactantes y siete en madres; mientras que por cada cinco embarazadas vacunadas se previno un caso de enfermedad respiratoria febril en madres o niños. Habría que vacunar a 16 embarazadas para prevenir un episodio documentado de influenza en niños.

\section{Conclusión}

La vacuna anti-Influenza redujo la enfermedad por influenza un
$63 \%$ en menores de seis meses y evitó un tercio de las enfermedades respiratorias febriles en embarazadas y lactantes. La vacunación anti-Influenza materna es una estrategia con sustanciales beneficios para las embarazadas y los niños.

Palabras claves: vacunación, embarazadas, lactantes, Influenza. Keywords: vaccination, pregnancy, infants, influenza.

Fuentes de financiamiento: Fundación Bill and Melinda Gates, U.S. Agency for International Development (USAID), Department of Health and Human Services, National Vaccine Program Office, Wyeth Pharmaceuticals, Thrasher Research Fund, Aventis Pasteur, Intemational Centre for Diarrheal Disease Research, Bloomberg School of Public Health at Johns Hopkins University.

\section{Comentario}

Las complicaciones asociadas a la infección por Influenza son más frecuentes en mujeres embarazadas'. En EEUU se recomienda la vacunación anti-Influenza en mujeres que están cursando el segundo y tercer trimestre de embarazo durante la estación invernal ${ }^{2}$. Este estudio es el primer ensayo clínico que intenta avalar la vacunación anti-Influenza en embarazadas, demostrando un efecto beneficioso en madres y niños. Sin embargo, tiene la limitación de que la intervención evaluada (vacunación anti-Influenza) no fue la única diferencia entre el grupo intervención y el grupo control ya que el grupo control recibió vacuna anti-Neumoco (no recibida por el grupo intervención) lo que podría llevar a susbestimar la verdadera eficacia de la vacunación anti-Influenza. Por otro lado, el intervalo de confianza del resultado primario es amplio, siendo además complicada la extrapolación de sus resultados a nuestro medio ya que fue llevado a cabo en una región subtropical en la que la incidencia de Influenza no tiene variación estacional.

\section{Conclusiones de la comentadora}

El estudio que hemos resumido aporta datos para recomendar la vacunación en embarazadas durante el tercer trimestre y muestra efectos beneficiosos en menores de seis meses. Sin embargo, no es suficiente para establecer una recomendación a nivel poblacional dado que no evaluó eventos de mayor impacto sanitario como mortalidad, hospitalizaciones o ausencia laboral; siendo también necesarios estudios de costo-efectividad de este tipo de intervenciones.

Carolina Carrara [ Servicio de Medicina Familiar y Comunitaria del Hospital Italiano de Buenos Aires. carolina.carrara@hospitalitaliano.org.ar ] Recibido el 30/11/08 y aceptado el 10/02/09.

Carrara C. Efectividad de la vacuna antiinfluenza en embarazadas y lactantes. Evid. Actual. Práct. Ambul; 12(1):7, Ene-Mar 2009. Comentado de: Zaman K y col. Effectiveness of Maternal Influenza Immunization in Mothers and Infants. N Engl J Med 2008; 359:1555-64. PMID: 18799552. Disponible en URL: http://content.nejm.org/cgi/reprint/359/15/1555.pdf (último acceso: 10/02/09).

\section{Referencia}

1.Smith $\mathrm{N}$ y col. Advisory Committee on Immunization Practices, Prevention and control of influenza: recommendations of the Advisory Committee on Immunization Practices (ACIP). MMWR Recomm Rep 2006; 55: RR-10:1-42. 